\title{
PEANUT SHAPED BARS
}

\author{
J. A. SELLWOOD \\ Department of Physics and Astronomy, Rutgers University \\ P O Box 849, Piscataway NJ 08855-0849, U.S.A.
}

ABSTRACT. Recent 3-D $N$-body simulations have shown that the bars formed through the well-known global instability of discs later acquire a pronounced peanut shape when seen from the side. This paper reports a new calculation of the phenomenon using a finer grid and a slightly different initial model. The final peanut shaped bar, when viewed from within the disc, has an appearance remarkably similar to that of the Milky Way bulge. When viewed as a distant edge-on galaxy, an observer would see cylindrical rotation in the "bulge".

\section{Model and Evolution}

Combes \& Sanders (1981), Combes et al. (1990) and Raha et al. (1991) have reported 3-D $N$-body simulations of barred disc galaxies in which the bar always acquired a peanut shape when viewed from the side. The new experiment described here started from a $Q=1.2$, axisymmetric Kuz'min-Toomre disc represented by $50 \mathrm{~K}$ particles containing $70 \%$ of the total mass; the remaining $30 \%$ is in a rigid Plummer sphere which has half the scale length of that of the disc. (The earlier experiments by Raha et al. showed that the behaviour studied here is not greatly affected by this rigid bulge/halo approximation.) The model was evolved using a 3-D Cartesian particle-mesh code having $127^{2} \times 31$ cubic grid cells (double the resolution used by Raha' et al.).

As expected, the model first formed a bar which then began to bend out of the plane through the fire-hose instability. After reaching a bend angle of some $20^{\circ}$, the bar abruptly regained symmetry about the disc plane, but was quite a bit fatter normal to the plane and noticeably shorter. The bar is thickest at its ends, giving it a pronounced peanut shape when viewed from the side, but the thickness of the disc at larger radii is not much affected by the bending of the bar.

\section{2. "Observations" of the Final Model}

The peanut shape of the object is visible from viewing angles in the plane which are greater than about $30^{\circ}$ to the bar major axis, but at even smaller angles, the

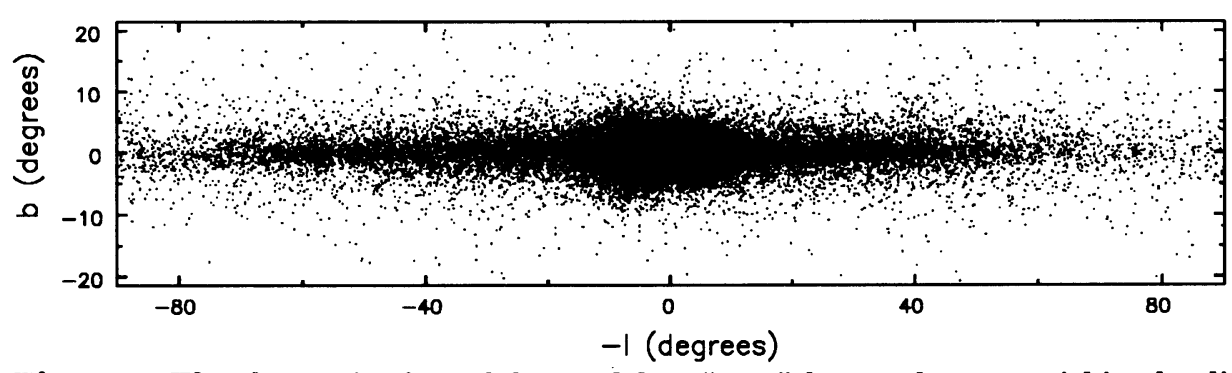

Figure 1 The sky projection of the model as "seen" by an observer within the disc looking towards the inner galaxy at an angle of $30^{\circ}$ to the bar major axis. 
object still appears boxy. Given the full 3-D spatial and velocity information, it is possible to "observe" the model from any arbitrary angle to deduce quantities to be compared with observational data.

\subsection{AS A MODEL OF THE MILKY WAY}

Figure 1 shows the sky projection of the model from a point at a distance from the centre approximately four times the bar semi-major axis, and at an angle of $30^{\circ}$ to that axis. This galactocentric distance was chosen such that the two lobes of the peanut subtend a longitudinal angle of approximately $10^{\circ}$. If the observing point is moved closer to the bar major axis the bulge loses some of its peanut appearance, becoming more box-like, and I would have to move the observing point inwards for the box feature to subtend the same angle; this would also magnify the longitudinal asymmetry. The resemblance to the bulge of the Milky Way is very striking, even the asymmetry in the $b$ extent of the bar between the near and far sides is comparable. Apart from obvious aspects such as the absence of obscuration in the model, the comparison could be improved still further by scaling the intensity of each point as the inverse square of its distance.

\subsection{AS A MODEL OF A DISTANT GALAXY}

Alternatively we can project the entire model to mimic the view from a great distance; the projected velocity field of the particles is shown in Figure 2. The absence of a dark halo does not affect the kinematics of the bulge region, but gives the model a slightly declining outer rotation curve which is somewhat unrealistic. One of the most striking features of Figure 2 is that the bulge-like object possesses apparent cylindrical rotation, as has been observed (e.g. Shaw et al. 1992).

\section{References}

Combes F \& Sanders R H, 1981. Astron. Astrophys. 86, 164.

Combes F, Debbasch F, Friedli D \& Pfenniger D, 1990. Astron. Astrophys. 233, 82.

Raha N, Sellwood J A, James R A \& Kahn F D, 1991. Nature 352, 411.

Shaw M, Wilkinson A \& Carter D, 1992. Preprint

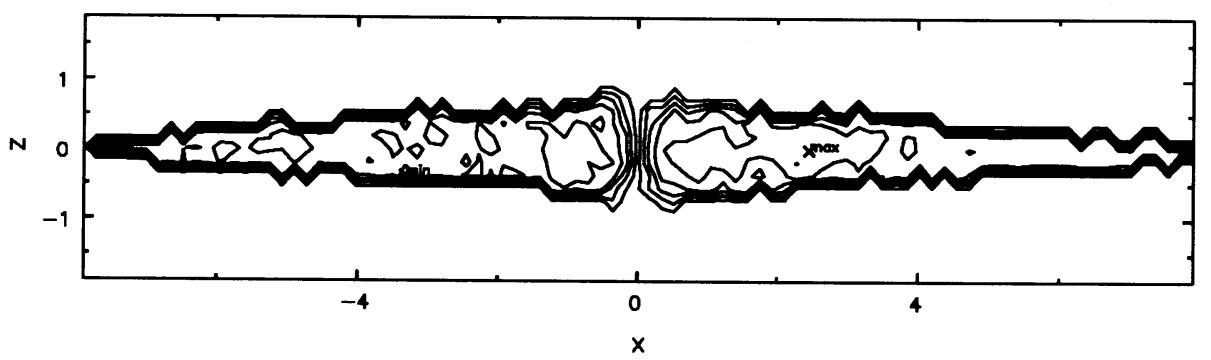

Figure 2 Line of sight streaming velocities of particles in the model (arbitrary scale) as seen by an observer at a great distance. The viewing angle is again from the mid-plane at an angle of $30^{\circ}$ to the bar major axis. The value of the streaming velocity has been set to zero in any bin containing fewer than 10 particles. 